\title{
Successful Closure of a Benign Refractory Tracheoesophageal Fistula Using an Over-the-Scope Clip after Failed Esophageal Stent Placement and Surgical Management
}

\author{
Nonthalee Pausawasdi ${ }^{1}$, Chotirot Angkurawaranon ${ }^{1}$, Tanyaporn Chantarojanasiri', Arunchai Chang ${ }^{1}$, Wanchai Wongkornrat ${ }^{2}$, \\ Somchai Leelakusolvong ${ }^{1}$ and Asada Methasate ${ }^{1,3}$ \\ ${ }^{1}$ Vikit Viranuvatti Siriraj Endoscopy Center, Faculty of Medicine Siriraj Hospital, Mahidol University, Bangkok, ${ }^{2}$ Division of Cardiothoracic \\ Surgery, Department of Surgery, Faculty of Medicine Siriraj Hospital, Mahidol University, Bangkok, ${ }^{3}$ Division of Minimally Invasive Surgery, \\ Faculty of Medicine Siriraj Hospital, Mahidol University, Bangkok, Thailand
}

Tracheoesophageal fistulas (TEFs) have traditionally been managed surgically, but the endoscopic approach is widely performed as a less invasive alternative. Different closure techniques have been proposed with inconsistent results. An over-the-scope clip (OTSC) appears to be a reasonable option, but long-term results have not been well defined. We report the long-term outcomes of a complex case of successful closure of a benign refractory TEF using an OTSC after failed surgical management and esophageal stent placement. Clin Endosc 2020;53:361-365

Key Words: Esophagorespiratory fistula; Over-the-scope clip; Tracheoesophageal fistula

\section{INTRODUCTION}

Tracheoesophageal fistulas (TEFs) can be caused by traumatic injury of the chest, surgical intervention, cancer, and post-radiation exposure. ${ }^{1,2}$ The common clinical manifestations are recurrent aspiration pneumonia, coughing after swallowing, and increased respiratory secretion. ${ }^{3}$ Diagnosis can be made by visualization of the fistula opening during esophagogastroduodenoscopy (EGD), bronchoscopy, contrast study, or computed tomography (CT). To avoid morbidity from surgical correction, endoscopic approaches using the injection of fibrin glue, suturing devices, esophageal stents,

Received: June 5, 2019 Revised: July 30, 2019

Accepted: August 4, 2019

Correspondence: Asada Methasate

Division of Minimally Invasive Surgery, Department of Surgery, Vikit Viranuvatti Siriraj Endoscopy Center, Faculty of Medicine Siriraj Hospital, Mahidol University, 2 Thanon Wang Lang, Khwaeng Siriraj, Bangkok 10700, Thailand Tel: +66-2-419-7282, Fax: +66-2-411-5013, E-mail: teamsster@gmail.com ORCID: https://orcid.org/0000-0002-8726-365X

(c) This is an Open Access article distributed under the terms of the Creative Commons Attribution Non-Commercial License (http://creativecommons.org/ licenses/by-nc/3.0) which permits unrestricted non-commercial use, distribution, and reproduction in any medium, provided the original work is properly cited. airway stents, through-the-scope (TTS) clips, and over-thescope clips (OTSCs) have been introduced. Esophageal stent placement has been the most commonly used endoscopic technique for TEF closure. ${ }^{4,5}$ The use of OTSCs has gained popularity in recent years; OTSCs can be used for the closure of entero-enteric fistulas and fistulas between the gastrointestinal tract and other organs such as the bronchus, skin, and urinary bladder. ${ }^{2}$ Nonetheless, fistula closure using OTSCs in benign refractory TEFs after failed esophageal stent placement and surgical management have not been reported. Herein, we report a case of successful OTSC placement for TEFs caused by trauma after failed esophageal stent placement and surgical management.

\section{CASE REPORT}

A 44-year-old male presented with aortic arch transection after a vehicular accident. He underwent a hemiarch replacement with innominate artery re-implantation. Two weeks later, he was noted to have recurrent aspiration pneumonia resulting in difficulty weaning off the ventilator and air leak- 
age from the chest tube, prompting further investigations. A CT scan of the chest revealed possible TEFs. Contrast study demonstrated a fistulous tract between the upper esophagus and left main bronchus. EGD revealed a 15-20 mm fistula opening located $26 \mathrm{~cm}$ from the incisors (Fig. 1A). Therefore, an $18 \times 120 \mathrm{~mm}$ Polyflex ${ }^{\mathrm{TM}}$ (Boston Scientific, Natick, MA, USA) esophageal stent was placed (Fig. 1B). Interval follow-up contrast studies showed persistent contrast leakage suggesting a non-healing fistula, and therefore, the stent was left in place for longer than expected. The stent was finally removed 9 months later due to a pressure effect to the left main bronchus causing left lung atelectasis and low-grade lung infection without resolution of the TEF. Despite prolonged stent place- ment, there was no epithelialization, granulation, or tissue overgrowth observed during stent removal. The stent was removed without difficulty using rat-tooth forceps. The patient then underwent surgery for fistula closure using Prolene 4/0 with intercostal muscle flap reinforcement.

Nonetheless, he continued to develop recurrent aspiration pneumonia, and an unresolved TEF was discovered despite attempted surgical repair. Thus, a fully covered $18 \times 120 \mathrm{~mm}$ Wallflex $^{\mathrm{TM}}$ (Boston Scientific) esophageal stent was placed. Follow-up endoscopy was performed after 6 months and showed a persistent TEF after stent removal; however, the size of the fistula opening decreased to less than $1 \mathrm{~cm}$ (Fig. 2).

At that time, the potential endoscopic treatment options
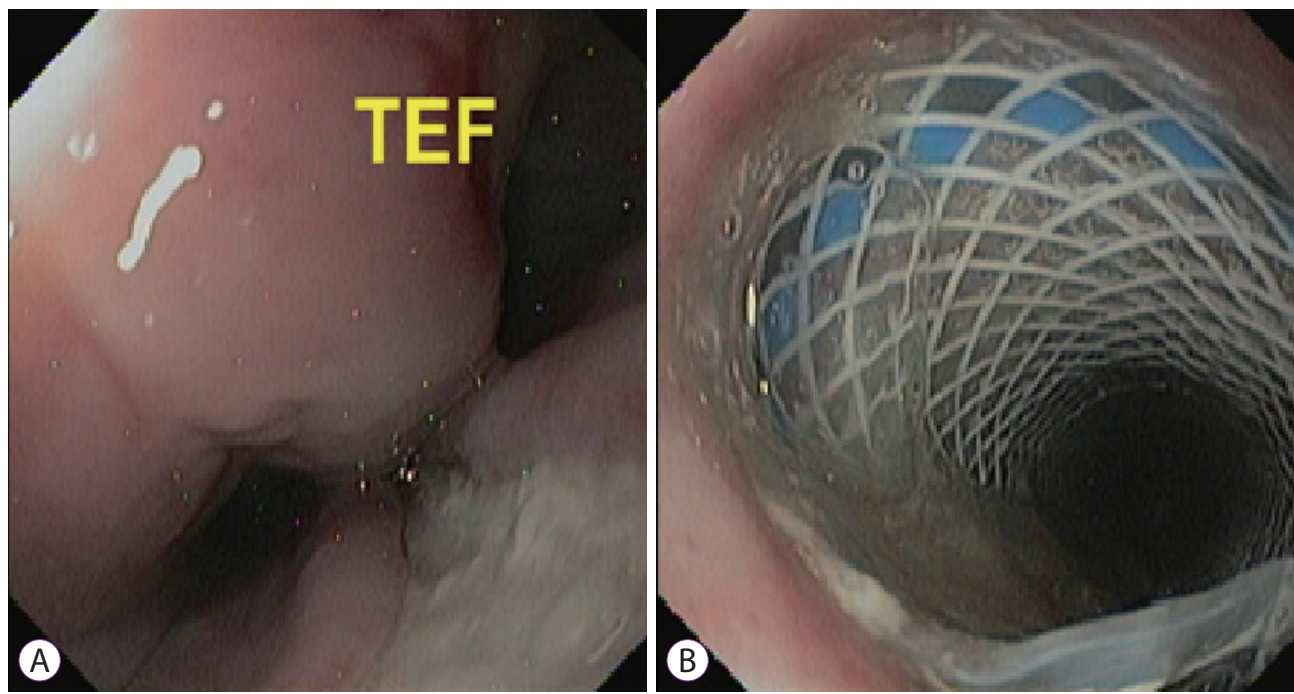

Fig. 1. (A) Endoscopic image demonstrates tracheoesophageal fistula (TEF) in the upper esophagus. (B) Placement of $18 \times 120 \mathrm{~mm}^{\text {Polyflex }}{ }^{\mathrm{TM}}$ (Boston Scientific, Natick, MA, USA) stent.
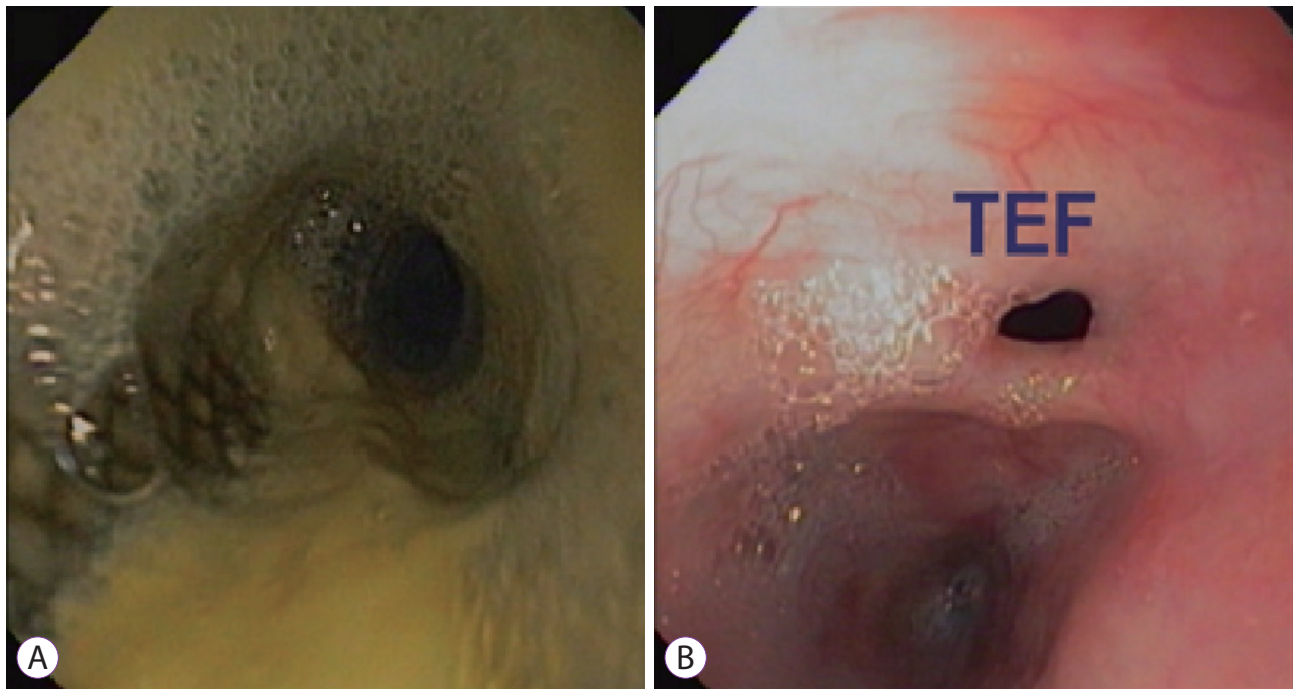

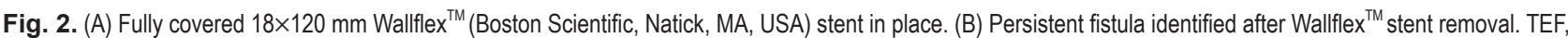
tracheoesophageal fistula. 
were OTSC and endoscopic vacuum-assisted closure (E-VAC). Endoscopic vacuum therapy is most commonly used for the treatment of esophagogastric anastomotic leaks in patients who failed to respond to esophageal stent placement, esophageal perforation, and postoperative leaks. However, data on E-VAC treatment of TEFs are limited, and a disadvantage of this technique is the need to repeat endoscopic procedures under anesthesia. ${ }^{6,7}$ Furthermore, the choice of endoscopic modality is often based on local expertise and readiness of devices. Therefore, we opted to use an OTSC (Ovesco Endoscopy, Tübingen, Germany) to close the fistula. Under endoscopy (Supplementary video 1), a TEF was located $26 \mathrm{~cm}$ from the incisors surrounded by fibrotic tissue. A $12 / 6 \mathrm{t}$ OTSC was affixed to the tip of a therapeutic gastroscope which was advanced to the fistula opening. Given the fibrotic nature of the edge of the fistula opening, a grasper was used to pull one end of the fistula opening while suctioning the surrounding tissue continuously into the cap. The clip was then deployed. We elected not to use argon plasma coagulation (APC) before applying the clip as it has been suggested that APC may not be beneficial when using an OTSC, given its transmural closure effect. Furthermore, thermal injury may cause tissue edema, making it more difficult to grasp the tissue into the cap. ${ }^{8}$

After confirming that the clip was in a good position, contrast injection was performed, which revealed no contrast leakage (Fig. 3). Follow-up esophagogram at 4 and 16 weeks
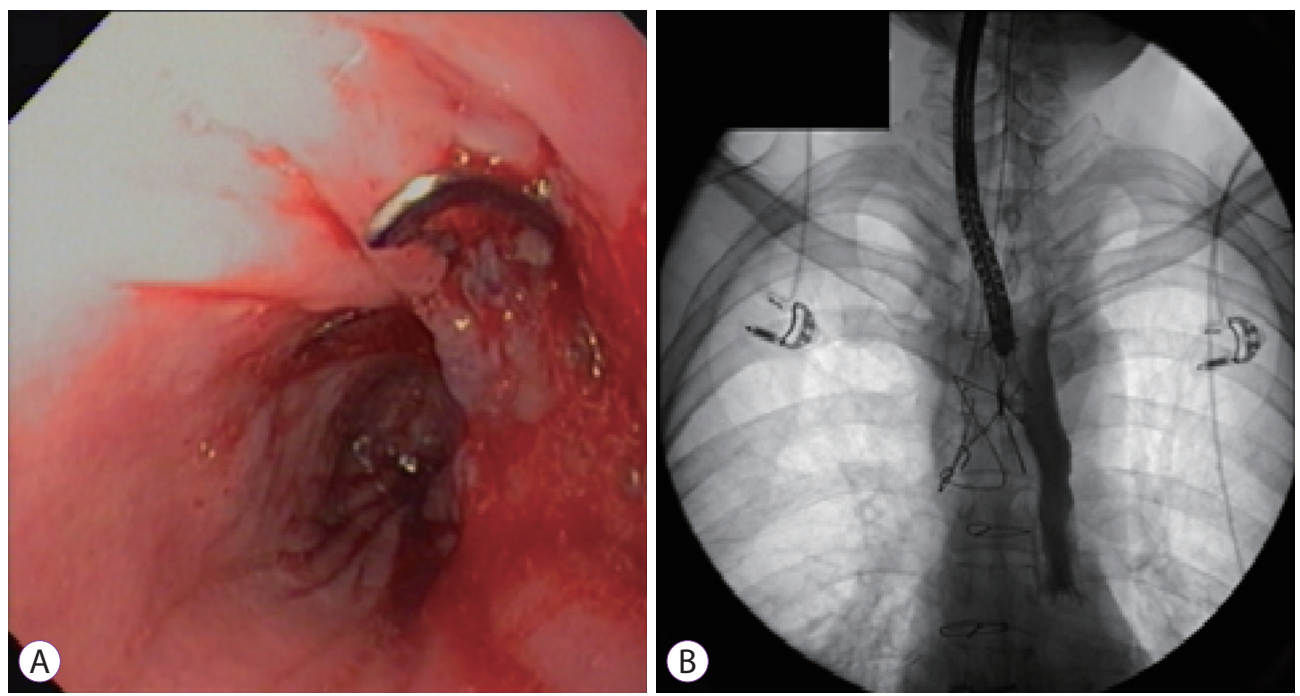

Fig. 3. (A) Endoscopic view of fistula closure with an over-the-scope clip. (B) Contrast injection under fluoroscopy shows no contrast leakage after placement of the clip
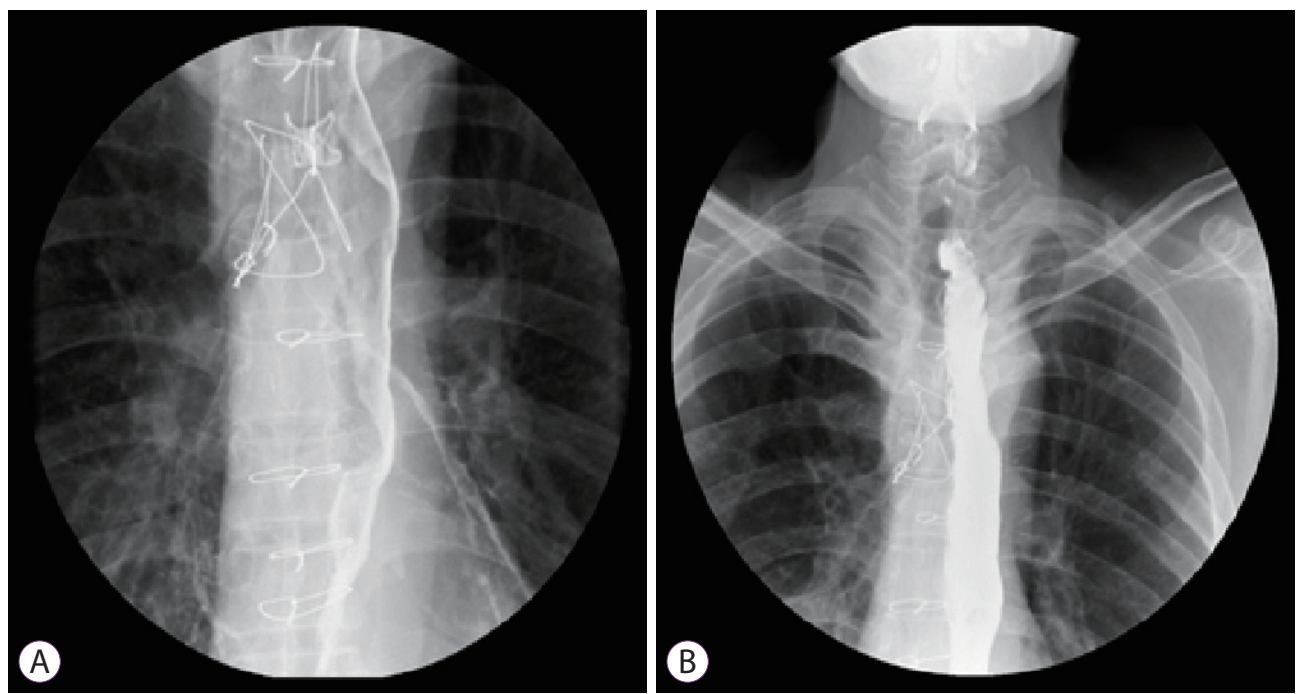

Fig. 4. Follow-up water-soluble esophagogram. (A) Contrast study performed at 4 weeks after treatment showing no leakage. (B) Contrast study performed at 16 weeks after treatment showing no leakage. 
showed no leakage (Fig. 4). The patient remained clinically well after 24 months of follow-up.

\section{DISCUSSION}

TEFs have been conventionally managed surgically, with a recent trend in practice toward endoscopic esophageal stent placement as it offers excellent technical success and less invasiveness. OTSCs for fistula closure have been introduced over the past decade but the data are still limited. Initial reports demonstrated promising technical success, but long-term outcomes are lacking. ${ }^{3,9,10}$ Subsequent retrospective studies assessing the clinical success of OTSCs for different indications revealed that this device was safe and effective in controlling gastrointestinal bleeding, perforation, and fistula closure; however, sufficient closure of chronic fistulas remained problematic and was associated with high recurrence rates. ${ }^{8,11}$ Difficult-to-treat fistulas in these series were reported to be enterocutaneous following percutaneous endoscopic gastrostomy (PEG)/jejunostomy (PEJ), post-radiation-related fistulas, and cancer-related fistulas. It is also important to mention subsequent fistula recurrence after an initial successful closure may occur despite the presence of the clip. Furthermore, an obvious fistulous tract had been observed adjacent to the OTSC on repeat endoscopy which may represent either clip migration from peristalsis or fistula recurrence. Given this situation, experts proposed that the definite closure of the fistula can only occur if the entire fistulous tract, including both the origin and insertion site, is treated. ${ }^{2} \mathrm{~A}$ recent multicenter prospective study emphasized the safety and efficacy of OTSCs for the treatment of anastomotic leakage after surgery. However, this method was less effective for chronic fistula closure compared to the treatment of perforation and leakage (25\% vs. $87 \%, p=0.025) .{ }^{12}$

A few retrospective cohort studies have evaluated long-term outcomes of OTSC fistula closure, and the results in all studies consistently showed a high rate of fistula recurrence ranging from $45 \%$ to $60 \%$ after 4 weeks. ${ }^{2,11,13-15}$ Clinical outcomes in a series including 47 patients after immediate successful fistula closure using an OTSC were as follows: fistula recurrence in $46 \%$ at 39 days and delayed clinical success in $53 \%$ at a median follow-up period of approximately 6 months. ${ }^{2}$ The etiologies of fistulas in this series were as follows: enterocutaneous following PEG, PEJ, surgery, and inflammatory process and gastro-gastric, colovesical, colovaginal, and tracheoesophageal following radiation therapy. To overcome the difficulty, an adjunctive method such as APC was proposed in order to denude the epithelialized tract in the hopes of promoting fistula closure. Nonetheless, the results did not show any added value. ${ }^{8}$
Recently, retrospective studies focusing on esophagorespiratory fistulas (ERFs) evaluated the factors influencing clinical outcomes after endoscopic treatment. ${ }^{4,5}$ Silon et al. ${ }^{4}$ recruited 25 patients undergoing 35 procedures, and $56 \%$ of the patients had malignant fistulas. The treatment modalities included placement of an esophageal fully covered-self-expandable-metallic stent (FCSEMS), combined esophageal stent and bronchial stent, combined esophageal stent and OTSC, and combined esophageal stents, OTSC, and additional methods such as TTS clip, endoscopic suture, and APC. The use of OTSC monotherapy was reported in one case of a benign fistula. The technical success was nearly $100 \%$ for all methods but overall clinical success was less than $50 \%$, with esophageal stent monotherapy having the best clinical success of $52 \%$. The study indicated that proximal ERFs were the most difficult to treat, with the highest rate of recurrent aspiration and minor adverse events. Despite overall suboptimal clinical success and survival, endoscopic therapy is considered a safe method and minimally invasive. In terms of endoscopic management of benign ERFs, a retrospective study of 22 patients was conducted to evaluate clinical outcomes and predictive factors associated with clinical success. ${ }^{5}$

The causes of ERFs in this series were surgical anastomotic fistulas, post-esophageal dilation, invasive ventilation, radiation therapy, and tracheostomy. The endoscopic method was selected based on the characteristics of the fistulas. An OTSC was selected for small fistula with smooth and non-fibrotic edges. For fistulas larger than 10-15 mm with fibrotic edges or associated stricture, a FCSEMS was used. Additional endoscopic methods such as APC may be applied at the endoscopist's discretion. The endoscopic success rate was reported to be $45.5 \%$, with a functional success rate of $55 \%$. Adverse events were reported in $40.9 \%$ of cases and included stent migration, bleeding, thoracic spondylodiscitis, food impaction, and major chest pain. The results demonstrated that the larger the fistula size, the more difficult to treat, and the chances of clinical success diminished dramatically if fistula healing did not occur after 6 months of treatment.

Taken together, the overall clinical success of endoscopic treatment for TEFs is about $50 \%$. The size and nature of the fistula appeared to be predictive factors of the outcomes. Nonetheless, the long-term data on the utility of OTSCs are scarce, and there has never been a report on endoscopic treatment options for TEFs caused by blunt chest injuries with failed esophageal stent placement and surgical management. In this report, we present a unique case of successful treatment of a benign refractory TEF, after sustained chest trauma from a motor vehicle accident, using OTSCs. We hypothesize that initial failure to close the fistula with stent placement was due to the large fistula size and friable surrounding tissue. 
Furthermore, the friability of esophageal tissue and on-going lung infection, along with the patient's suboptimal nutritional status at the time of surgery, may have led to disappointing results after surgical repair owing to poor tissue healing. Nevertheless, the size of the fistula opening became much smaller $(<1 \mathrm{~cm})$ with the fibrotic edge, and the surrounding tissue was no longer friable; consequently, OTSCs for fistula closure at this time were a reasonably good option. The fistula closure occurred within a few weeks after the procedure, and the patient remained clinically stable after 2 years of follow-up.

In conclusion, an OTSC can be a useful device for the treatment of a complex and refractory chronic TEF in select circumstances. The importance of the characteristics and nature of the fistula, along with proper patient selection, should be emphasized before choosing the endoscopic method for fistula closure.

Conflicts of Interest

The authors have no financial conflicts of interest.

\section{Supplementary Material}

Video 1. Endoscopic closure of a refractory tracheoesophageal fistula using an over-the-scope clip (https://doi.org/10.5946/ce.2019.106.v001).

\section{REFERENCES}

1. Santosham R. Management of acquired benign tracheoesophageal fistulae. Thorac Surg Clin 2018;28:385-392.

2. Law R, Wong Kee Song LM, Irani S, Baron TH. Immediate technical and delayed clinical outcome of fistula closure using an over-the-scope clip device. Surg Endosc 2015;29:1781-1786.
3. So BJ, Adler DG. Closure of a chronic, non-healing tracheoesophageal fistula with a new over-the-scope clip. ACG Case Rep J 2014;2:18-20.

4. Silon B, Siddiqui AA, Taylor LJ, Arastu S, Soomro A, Adler DG. Endoscopic management of esophagorespiratory fistulas: a multicenter retrospective study of techniques and outcomes. Dig Dis Sci 2017;62:424-431.

5. Debourdeau A, Gonzalez JM, Dutau H, Benezech A, Barthet M. Endoscopic treatment of nonmalignant tracheoesophageal and bronchoesophageal fistula: results and prognostic factors for its success. Surg Endosc 2019;33:549-556.

6. Bemelman WA, Baron TH. Endoscopic management of transmural defects, including leaks, perforations, and fistulae. Gastroenterology 2018;154:1938-1946.e1.

7. Bludau M, Fuchs HF, Herbold T, et al. Results of endoscopic vacuum-assisted closure device for treatment of upper GI leaks. Surg Endosc 2018;32:1906-1914.

8. Baron TH, Song LM, Ross A, Tokar JL, Irani S, Kozarek RA. Use of an over-the-scope clipping device: multicenter retrospective results of the first U.S. experience (with videos). Gastrointest Endosc 2012;76:202-208.

9. Zolotarevsky E, Kwon Y, Bains M, Schattner M. Esophagobronchial fistula closure using a novel endoscopic over-the-scope-clip. Ann Thorac Surg 2012;94:e69-e70.

10. Traina M, Curcio G, Tarantino I, et al. New endoscopic over-the-scope clip system for closure of a chronic tracheoesophageal fistula. Endoscopy 2010;42 Suppl 2:E54-E55.

11. Kirschniak A, Subotova N, Zieker D, Konigsrainer A, Kratt T. The overthe-scope clip (OTSC) for the treatment of gastrointestinal bleeding, perforations, and fistulas. Surg Endosc 2011;25:2901-2905.

12. Lee HL, Cho JY, Cho JH, et al. Efficacy of the over-the-scope clip system for treatment of gastrointestinal fistulas, leaks, and perforations: a Korean multi-center study. Clin Endosc 2018;51:61-65.

13. Albert JG, Friedrich-Rust M, Woeste G, et al. Benefit of a clipping device in use in intestinal bleeding and intestinal leakage. Gastrointest Endosc 2011;74:389-397.

14. von Renteln D, Denzer UW, Schachschal G, Anders M, Groth S, Rösch T. Endoscopic closure of GI fistulae by using an over-the-scope clip (with videos). Gastrointest Endosc 2010;72:1289-1296.

15. Mercky P, Gonzalez JM, Aimore Bonin E, et al. Usefulness of overthe-scope clipping system for closing digestive fistulas. Dig Endosc 2015;27:18-24. 